\title{
What is behind the plastic strain rate?
}

\section{Journal Article}

Author(s):

Hütter, Markus; Grmela, Miroslav; Öttinger, Hans Christian (1)

Publication date:

2009-08

Permanent link:

https://doi.org/10.3929/ethz-b-000021240

Rights / license:

In Copyright - Non-Commercial Use Permitted

Originally published in:

Rheologica Acta 48(7), https://doi.org/10.1007/s00397-009-0371-y 


\title{
What is behind the plastic strain rate?
}

\author{
Markus Hütter • Miroslav Grmela • \\ Hans Christian Öttinger
}

Received: 25 February 2009 / Accepted: 31 May 2009 / Published online: 17 June 2009

(C) Springer-Verlag 2009

\begin{abstract}
The plastic strain rate plays a central role in macroscopic models on elasto-viscoplasticity. In order to discuss the concept behind this quantity, we propose, first, a kinetic toy model to describe the dynamics of sliding layers representative of plastic deformation of single crystalline metals. The dynamic variable is given by the distribution function of relative strains between adjacent layers, and the plastic strain rate emerges as the average hopping rate between energy wells. We demonstrate the behavior of this model under different deformations and how it captures the elastic-to-plastic transition. Second, the kinetic toy model is reduced to a closed evolution equation for the average of the relative strain, allowing us to make a direct link to macroscopic theories. It is shown that the constitutive relation for the plastic strain rate does not only depend on the stress, but also on the macroscopic applied deformation rate, contrary to common practice.
\end{abstract}

Keywords Plasticity Plastic strain rate $\cdot$ Reference state $\cdot$ Kinetic model $\cdot$ Constitutive relation . Reduced description

M. Hütter $(\bowtie) \cdot$ H. C. Öttinger

Department of Materials, Polymer Physics, ETH Zürich,

Wolfgang-Pauli-Str. 10, 8093 Zürich, Switzerland

e-mail: markus.huetter@mat.ethz.ch

M. Grmela

Department of Chemical Engineering,

École Polytechnique de Montréal,

H3C 3A7, Montréal, Quebec, Canada

\section{Introduction}

In macroscopic approaches of plasticity theory, the distinction between the initial state of deformation, current state, and intermediate stress-free state, reached from the current state upon cessation of the applied stress, is at the heart of all efforts (Besseling and van der Giessen 1993). Different formulations of plasticity exist depending on whether a static yielding criterion is invoked. In the absence of such, a measure for the elastic part of deformation is used as the dynamic "structural" variable of the material. For this variable, one invents irreversible dynamics in order to account for the rate-dependent plasticity (Boyce et al. 1988; Tervoort et al. 1998; Hütter and Tervoort 2008b), based on the seminal work of Leonov (1976). While the elastic left Cauchy-Green tensor is the most common choice for the dynamic variable, it has been argued recently that the elastic part of the deformation gradient $\boldsymbol{F}, \boldsymbol{F}^{\mathrm{e}}$, is a more suitable dynamic variable in an Eulerian formulation when dealing with general anisotropic materials (Hütter and Tervoort 2008a, b, c). In a macroscopic flow field $\boldsymbol{v}(\boldsymbol{r})$, the evolution equation can be written in the form (see also Rubin $(1994,1996)$ for a similar approach)

$\frac{D}{D t} \boldsymbol{F}^{\mathrm{e}}=\left(\boldsymbol{\kappa}-\boldsymbol{\kappa}^{\mathrm{p}}\right) \cdot \boldsymbol{F}^{\mathrm{e}}$,

with the material derivative $D / D t=\partial / \partial t+v \cdot \nabla$ and the transposed velocity gradient $\kappa=(\nabla v)^{T}$. The symbol $\kappa^{\mathrm{p}}$ stands for the so-called plastic deformation gradient, for which a constitutive relation is required. In purely macroscopic theories of crystalline metals, slip systems, indexed with $i$, with slip directions $\boldsymbol{s}_{i}$ and slip plane normal $\boldsymbol{n}_{i}$ capture the key modes of deformation. 
If $\dot{\gamma}_{\mathrm{p}, i}$ denotes the rate of plastic deformation in slip system $i$, the generic ansatz for the plastic deformation gradient tensor is given by

$\boldsymbol{\kappa}^{\mathrm{p}}=\sum_{i} \dot{\gamma}_{\mathrm{p}, i} \boldsymbol{s}_{i} \otimes \boldsymbol{n}_{i}$,

where the summation runs over all active slip systems (Asaro 1983). In the absence of detailed dislocation dynamics, it is usually assumed that $\dot{\gamma}_{\mathrm{p}, i}$ depends on the state of deformation through the Cauchy stress tensor $\sigma$. Particularly, $\dot{\gamma}_{\mathrm{p}, i} \propto \tau_{i}^{k}$ with resolved shear stress $\tau_{i}=\boldsymbol{s}_{i} \cdot \boldsymbol{\sigma} \cdot \boldsymbol{n}_{i}$ on glide system $i$ (Asaro 1983; Pan and Rice 1983). If the dynamics of dislocations are included in the full set of evolution equations, the dislocation densities enter into $\dot{\gamma}_{p, i}$ as well (Asaro 1983; Hutchinson 1976; Pan and Rice 1983; Estrin 1996; Tabourot et al. 1997; Schulze and Vöhringer 2001). The evolution equation (Eq. 1) for the elastic part of the deformation gradient can, in principle, be used to describe both the elasto-viscoplasticity of solids and the viscoelasticity of liquids, depending on the constitutive relation for $\boldsymbol{\kappa}^{\mathrm{p}}$. The main difference is that, in elasto-viscoplasticity, the plastic deformation gradient is a strong function of the applied stress, leading to a rather abrupt change from solid- to fluid-like behavior.

Our primary interest is in discussing some issues about the emergence of plastic deformation upon increasing the stress level, i.e., about the background behind a macroscopic theory as described above in Eq. 1.
To that end, we restrict ourselves to shear deformations in the $x y$-plane exclusively,

$\boldsymbol{\kappa}=\left(\begin{array}{ccc}0 & \dot{\gamma}_{\mathrm{m}} & 0 \\ 0 & 0 & 0 \\ 0 & 0 & 0\end{array}\right), \quad \boldsymbol{F}^{\mathrm{e}}=\left(\begin{array}{ccc}1 & F_{x y}^{\mathrm{e}} & 0 \\ 0 & 1 & 0 \\ 0 & 0 & 1\end{array}\right)$,

with shear rate $\dot{\gamma}_{\mathrm{m}}$; the subscript "m" is used for later convenience to indicate that this is a "macroscopic" shear rate. The evolution equation (Eq. 1) implies that $\boldsymbol{\kappa}^{\mathrm{p}}$ is of the same tensorial form as $\boldsymbol{\kappa}$, with $\dot{\gamma}_{\mathrm{m}}$ replaced by $\dot{\gamma}_{p}$. In view of Eq. 2, this means that we consider situations with only a single slip system active, with $s$ and $\boldsymbol{n}$ being the $x$ - and $y$-directions, respectively. For these circumstances, Eq. 1 reduces to

$\frac{D}{D t} F_{x y}^{\mathrm{e}}=\dot{\gamma}_{\mathrm{m}}-\dot{\gamma}_{\mathrm{p}}$,

with $\dot{\gamma}_{\mathrm{p}}$ the plastic strain rate. In this special case, the task of modeling consists in finding a constitutive relation for $\dot{\gamma}_{\mathrm{p}}$.

The top part of Fig. 1 shows schematically different levels of description of crystalline metals, from left to right: single atoms, dislocation lines and slip planes, elasticity and plasticity with deformation tensors, and the continuum material. For comparison, possible different levels of description for polymeric liquids are depicted as well: chains as composed of atoms, beadspring chain with (usually purely entropic) springs, Maxwell model with a conformation tensor, and the continuum description (Bird et al. 1987; Larson 1999).
Fig. 1 Schematic representation of the multiple levels of description of crystalline metals and polymers
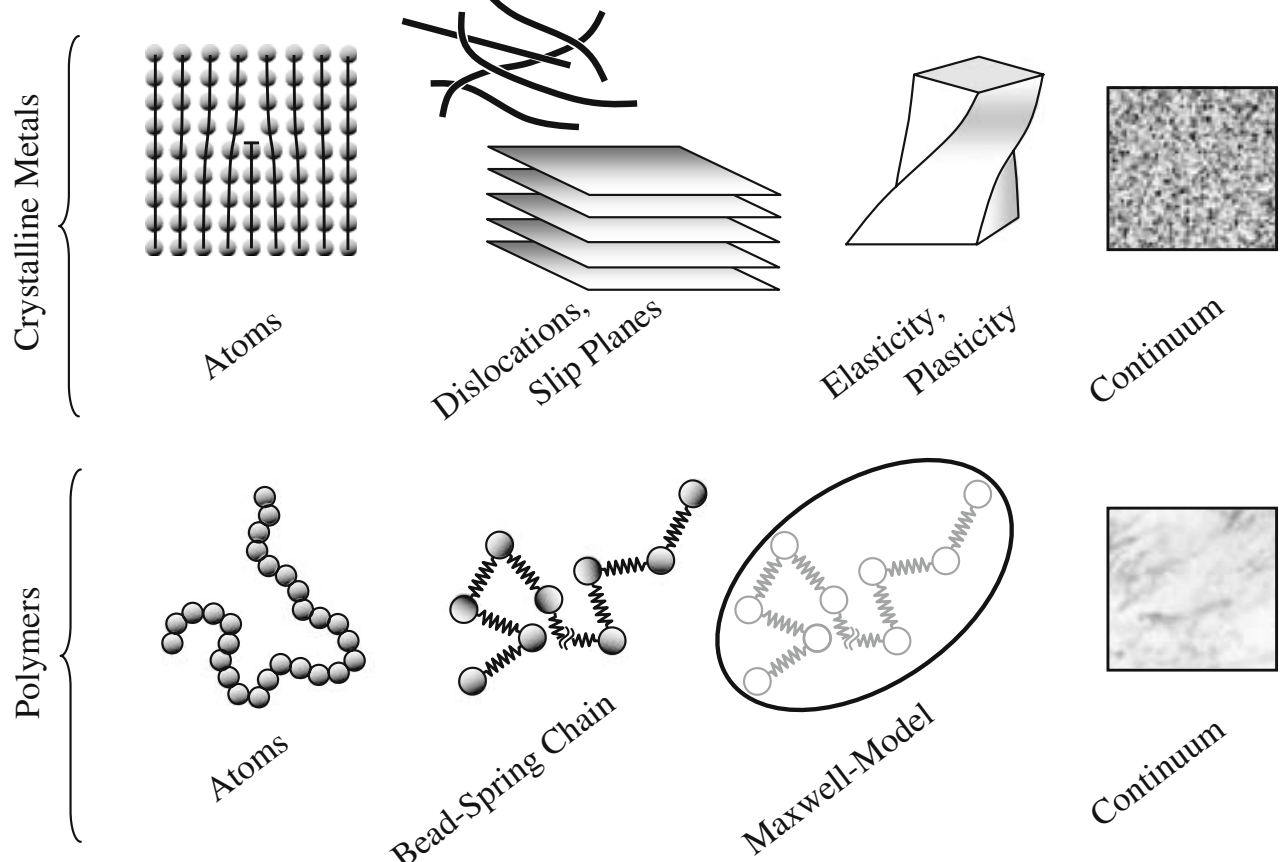
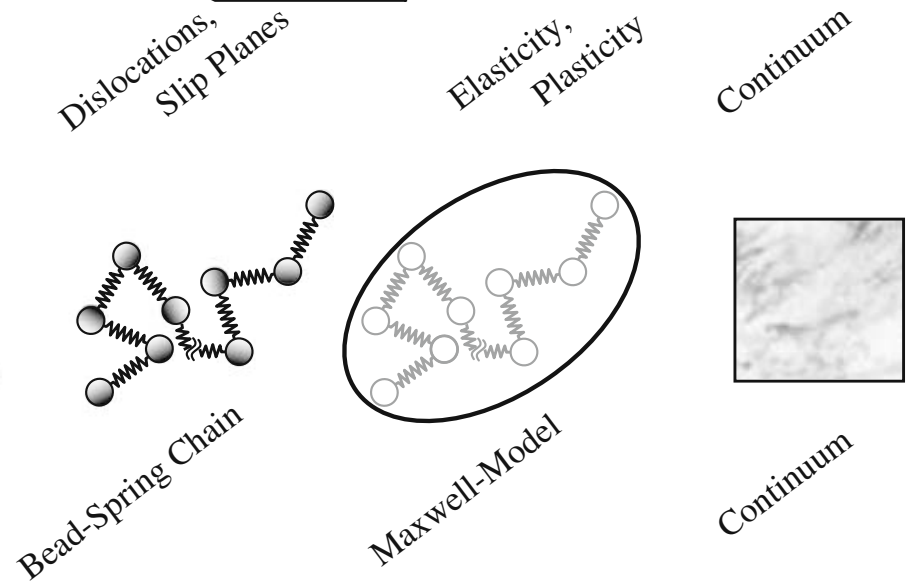
In order to discuss the meaning of the plastic strain rate $\dot{\gamma}_{\mathrm{p}}$, it is useful to relate the macroscopic description to one of the more fine-grained ones. For example, it has been shown that $\dot{\gamma}_{p}$ is related to the time-correlation of the fluctuations in the deformation gradient if the latter is expressed in terms of the rapidly moving microscopic constituent particles (Hütter and Tervoort 2008c). Conversely, one can choose to link $\dot{\gamma}_{\mathrm{p}}$ to the dynamics of dislocations (Orowan 1934; Von Polanyi 1934; Taylor 1934), as done to explain work-hardening by moving mutually interacting dislocations (Kocks 1976; Mecking and Kocks 1981; Kuhlmann-Wilsdorf 1985; Kocks and Mecking 2003). The link between the dislocation picture and a macroscopic description is established through the Orowan relation (Asaro 1983; El-Azab 2000; Groma et al. 2003; Arsenlis et al. 2004). Here, in contrast, we follow an alternative route. Namely, we study the dynamics of sliding layers, with the important main advantage that this will allow us eventually to connect in a direct and clean fashion to the macroscopic modeling approaches as the one specified in Eq. 1. Specifically, we introduce a kinetic toy model that encompasses both the elastic deformation and the aspect of plastic flow in the deformation of crystalline metals, thereby also enabling the modeling of the elastic-toplastic transition.

The manuscript is organized as follows: After formulating the kinetic toy model in the section "Formulation of the kinetic toy model," we illustrate with certain examples the predictions for the model under steady and transient conditions in the section "Model predictions." In the section "Reduction to a closed firstmoment description," the kinetic model is then reduced to a closed-form evolution equation for the average strain, which can be identified with the component $F_{x y}^{\mathrm{e}}$ in Eq. 3. In the section "Discussion," the results are discussed and the relation of the proposed kinetic toy model to the concept of dislocations is illustrated.

\section{Formulation of the kinetic toy model}

The most fundamental ingredient for the model presented here is that there is an energetically favored configuration, the system can be driven out of by external action to fall into an other equivalent favorable configuration. "Equivalent" here means that the energetics around all favorable configurations look identical, and the rate of transition is the only measure of non-elastic (i.e., plastic) deformation. This scenario is realized in single-crystalline metals in the most pure form. For this case, the toy model can be used to describe the microscopic kinetics of crystal plasticity by sliding lay- ers. However, despite the repeated mention of metal plasticity throughout the paper, one must keep in mind that this application of the model is not exclusive. For example, it is possible that the model is applicable to the motion of defects that cause amorphous solid polymers to behave viscoplastically, a point on which we will comment in the "Discussion" section. Regardless of the specific application, it can be said that, in general, the (resolved) shear stress is the cause of plastic deformation (Callister 2000; Haasen 1978). Therefore, we will restrict ourselves to shear deformations in the following.

Let us consider a stack of crystal layers. The instantaneous arrangement within that stack can be captured by the relative shear strains between any two adjacent layers, $\gamma$. Two adjacent layers interact by a potential $\Phi(\gamma)$ that reflects the periodicity of the crystal and its preferred equilibrium structure. More precisely, for two layers displaced by $\gamma$ with respect to each other, $\Phi(\gamma)$ summarizes all microscopic interaction energies within the volume $V$ of one crystal unit cell, divided by $V$, i.e., $\Phi$ has units of an energy density. By definition, the energy minimum shall occur for $\gamma=0$, corresponding to the equilibrium crystal structure. Due to the crystal symmetry, the potential $\Phi$ is symmetric around $\gamma=0$. The periodicity is denoted by $\Gamma>0$, while, for later convenience, $\Delta_{\Gamma}=[-\Gamma / 2, \Gamma / 2]$ is introduced to denote the $\gamma$ interval, which is bound on both sides by the location of the maximum in $\Phi$. A prototype example for $\Phi$ is

$\Phi(\gamma)=\frac{\Phi_{0}}{2}\left(1+\cos \left(2 \pi \frac{\gamma+\Gamma / 2}{\Gamma}\right)\right)$,

which vanishes at $\gamma=0$ and has a maximum of $\Phi_{0}$ at $\gamma= \pm \Gamma / 2$.

The time-dependent distribution function of the strains is denoted by $p(\gamma, t)$, and will serve as the dynamic variable in the proposed kinetic theory model for crystal plasticity. However, we begin with discussing the dynamics of the strain variable $\gamma$ itself, which must account for the following effects. Under the application of a small shear stress, the material deforms elastically, i.e., the deformation is recoverable. Here, "small shear stress" implies that the barriers between wells in the periodic potential $\Phi(\gamma)$ cannot be surmounted. Upon exerting a large enough shear stress, however, the energetic hindrance is overcome and the crystal planes begin to move relative to each other into the next energy well, i.e., plastic irreversible flow sets in. In addition, one must account for the fact that a layer within a single crystalline grain is made of a large number of microscopic particles, and hence, the layer as an entity experiences irregular thermal motion at finite 
temperature. After neglecting inertia of the sliding layers, the evolution of $\gamma$ can thus be described by the stochastic differential equation (Gardiner 1985; Öttinger 1996)

$d \gamma=\frac{1}{\hat{\eta}}\left(\sigma_{0}-\frac{\partial \Phi}{\partial \gamma}\right) d t+\sqrt{2 D} d W_{t}$,

where $\hat{\eta}$ is a friction coefficient. The prefactor $\sqrt{2 D}$ in the noise term represents the strength of fluctuations, with $d W_{t}$ as the random increment of a stochastic Wiener process with statistical averages $\left\langle d W_{t}\right\rangle=0$ and $\left\langle d W_{t} d W_{t^{\prime}}\right\rangle=\delta\left(t-t^{\prime}\right) d t$. In addition to the force due to the symmetric crystal potential, an "external" parameter $\sigma_{0}$ has been introduced, in order to represent the driving of the system out of equilibrium by the applied stress.

While, as a result of the applied stress, the transition of $\gamma$ from one energy well to another one in the periodic energy landscape signifies plastic flow, the cessation of that stress will prevent $\gamma$ from going back to the original well. There are two possibilities to interpret $\gamma$ and to use the evolution equation (Eq. 5). Either $\gamma$ is taken as the total strain, or it is interpreted as measuring that part of the strain that is recovered upon cessation of the applied stress, i.e., capturing the relative distance between the current state and the closest stress-free reference state. In our model, we adopt the latter interpretation, which is represented in Eq. 5 by requiring that $\gamma$ can only take values in the previously defined domain $\Delta_{\Gamma}$, and by imposing periodic boundary conditions. However, this necessitates keeping track of the rate of transitions between different wells in the energy landscape, on which we will comment further below.

The stochastic differential equation (Eq. 5) can equivalently be represented in terms of the FokkerPlanck equation for the time-dependent probability distribution function $p(\gamma, t)$ (Gardiner 1985; Öttinger 1996),

$\frac{\partial p}{\partial t}=-\frac{\partial j}{\partial \gamma}$,

with the probability current

$j=\frac{1}{\hat{\eta}}\left(\sigma_{0}-\frac{\partial \Phi}{\partial \gamma}\right) p-D \frac{\partial p}{\partial \gamma}$,

where it has been assumed that the diffusion coefficient $D$ does not depend on $\gamma$. Requiring that the distribution of strains at equilibrium in the absence of external driving $\left(\sigma_{0}=0\right)$ be given by the Boltzmann distribution, $p_{\text {eq }}(\gamma)=\mathcal{N}^{-1} \exp (-\Phi(\gamma) / \theta)$ with the thermal energy per crystal unit cell $\theta=k_{\mathrm{B}} T / V$ and normalization constant $\mathcal{N}$, leads to the fluctuation-dissipation relation between the friction and diffusion coefficients (Kubo 1966; Kubo et al. 1985)

$D=\theta / \hat{\eta}$

In analogy to Eq. 5, Eq. 6 must be solved on the interval $\Delta_{\Gamma}$ with periodic boundary conditions.

With the Fokker-Planck equation (Eq. 6) for the evolution of the distribution function $p(\gamma, t)$, we are in the position to give a transparent interpretation of the transition between the different energy wells. According to Eqs. 5 and 6, due to the driving parameter $\sigma_{0}>0\left(\sigma_{0}<0\right), \gamma$ has a tendency to cross the boundary of the interval $\Delta_{\Gamma}$ more often in the positive (negative) direction, which is represented on the level of the distribution function as a non-vanishing current density $j$ at the domain boundary, $j(-\Gamma / 2)=j(\Gamma / 2) \neq 0$. Upon leaving the interval $\Delta_{\Gamma}$ at one end and entering at the other end by way of the periodic boundary conditions, a shift between adjacent layers by one lattice unit has occurred, representative of plastic flow. Therefore, a meaningful definition of the plastic strain rate $\dot{\gamma}_{p}$ is given by the probability current density at the boundary of the $\gamma$-domain multiplied by $\Gamma$,

$\dot{\gamma}_{\mathrm{p}} \equiv \Gamma j(\gamma=\Gamma / 2)$,

both in stationary and transient situations. It serves as a counter for how many sliding events take place per unit time, which is needed in order to relate to the macroscopic applied shear rate, as discussed in the section "Model predictions."

While the periodicity is a crucial ingredient in the proposed model, it is to be clearly distinguished from seemingly similar approaches in the rheology literature. For example, in the reptation model of polymer rheology or in the description of axisymmetric rigid particles, the distribution function of an orientation vector of unit length is studied (Bird et al. 1987; Larson 1999; Öttinger 1996; Kröger 2005). There, the mathematical requirement of periodicity represents the identification of states that are identical in every respect. However, in the model proposed here, the periodicity represents the notion of equivalent (reference) states, that differ by intermediate hopping events, highlighting the need to have access to the average transition rate by way of Eq. 8, i.e., to the plastic rate of deformation.

In order to study the mechanical behavior of the kinetic toy model, a proper definition of the shear stress must be established. To achieve that goal, one can proceed by taking the definition of the shear stress $\sigma$, i.e., by considering forces across a test plane parallel to 
the crystal planes, and then looking at their components parallel to the plane. As a result, one finds

$\sigma \equiv\left\langle\frac{\partial \Phi}{\partial \gamma}\right\rangle$

in analogy to Kramers-type expressions in polymer kinetic theory. We mention in passing that the expression Eq. 9 for the shear stress can also be derived by invoking nonequilibrium thermodynamics techniques, e.g., Beris and Edwards (1994), Grmela and Öttinger (1997), Öttinger and Grmela (1997), Öttinger (2005). There, it is recognized that the expression for the stress tensor and the dynamics of the structural variables, here $p(\gamma, t)$, are two closely related model ingredients. The identification (Eq. 9) is also supported by continuum approaches to finite elasticity. Evaluating the general expression for the Cauchy stress tensor for finite elasticity in pure shear deformation (Truesdell and Noll 1992), the shear stress is found to be given by the derivative of the specific energy density with respect to the shear strain.

With the aid of the diffusion equation (Eq. 6), the shear stress (Eq. 9) for general transient situations can be written in the form

$\sigma=\sigma_{0}-\hat{\eta} \dot{\gamma}_{\mathrm{m}}$,

with the macroscopic shear rate defined as

$\dot{\gamma}_{\mathrm{m}}=\frac{d}{d t}\langle\gamma\rangle+\dot{\gamma}_{\mathrm{p}}$.

This definition of $\dot{\gamma}_{\mathrm{m}}$ represents the decomposition of the macroscopic shear rate into, first, the rate of average deformation with $\gamma \in \Delta_{\Gamma}$ and, second, the shear rate due to irreversible sliding by entire lattice units. Correspondingly, one should note the difference in the definitions of $d\langle\gamma\rangle / d t$ and $\dot{\gamma}_{\mathrm{p}}$ given in terms of a usual average over the interval $\Delta_{\Gamma}$ and an unconventional current density across the domain boundary, respectively. The derivation of $\dot{\gamma}_{p}$ from the kinetic model is a major result of the paper, as it describes the transition rate between equivalent discrete reference states. Note that, according to Eq. 10a, the formal driving parameter $\sigma_{0}$ can be expressed purely in terms of macroscopically measurable quantities, namely, the macroscopic shear rate $\dot{\gamma}_{\mathrm{m}}$ and the shear stress $\sigma$.

Two specific points are noteworthy. First, the definitions of the shear stress (Eq. 9) and, particularly, the macroscopic shear rate (Eq. 10b) are supported by a description that does not consider the reduced space $\gamma \in \Delta_{\Gamma}$ together with the periodicity condition on $p(\gamma, t)$, but rather discusses the unbound $\gamma$-domain and distinguishes different reference states (Öttinger, unpublished manuscript). Second, and even more im- portantly, Eq. 10b agrees with Eq. 3b, since the average elastic strain $\langle\gamma\rangle$ is identical to the component $F_{x y}^{\mathrm{e}}$ of the macroscopic deformation gradient. However, for given $\dot{\gamma}_{\mathrm{m}}$, Eq. $10 \mathrm{~b}$ is rather a physical relation between two functionals of the distribution function $p(\gamma, t)$, namely between $\langle\gamma\rangle$ and $\dot{\gamma}_{\mathrm{p}}$. The interpretation of Eq. $10 \mathrm{~b}$ as a (closed) evolution equation for $\langle\gamma\rangle$ is only possible after a reduction step is performed; see the section "Reduction to a closed first-moment description."

\section{Model predictions}

\section{Stationary situations}

The stationary solution to the diffusion equation (Eq. 6) can be determined for constant driving parameter $\sigma_{0}$. Using the abbreviation $\tilde{\Phi}\left(\gamma ; \sigma_{0}\right)=(\Phi(\gamma)-$ $\left.\sigma_{0} \gamma\right) / \theta$, one finds

$$
\begin{aligned}
p\left(\gamma ; \sigma_{0}\right)= & \frac{1}{\mathcal{N}\left(\sigma_{0}\right)} e^{-\tilde{\Phi}\left(\gamma ; \sigma_{0}\right)} \\
& \times\left[1-c\left(\sigma_{0}\right) \int_{-\Gamma / 2}^{\gamma} e^{\tilde{\Phi}\left(\gamma^{\prime} ; \sigma_{0}\right)} d \gamma^{\prime}\right],
\end{aligned}
$$

with normalization constant $\mathcal{N}$ such that $\int_{-\Gamma / 2}^{\Gamma / 2} p(\gamma) d \gamma=1$, and $c$ independent of $\gamma$. From the periodicity condition $p\left(-\Gamma / 2 ; \sigma_{0}\right)=p\left(\Gamma / 2 ; \sigma_{0}\right)$, one obtains

$c\left(\sigma_{0}\right)=\frac{1-e^{-\Gamma \sigma_{0} / \theta}}{\int_{-\Gamma / 2}^{\Gamma / 2} e^{\tilde{\Phi}\left(\gamma^{\prime} ; \sigma_{0}\right)} d \gamma^{\prime}}$.

As the probability current $j$ for the stationary solution (Eq. 11) assumes the form $j_{\mathrm{st}}=D c / \mathcal{N}$ and in view of the plastic strain rate (Eq. 8), the expression Eq. $11 \mathrm{~b}$ can be used to discuss the relation between the stationary plastic strain rate $\dot{\gamma}_{\mathrm{p}, \mathrm{st}}$ and the driving parameter $\sigma_{0}$. We get $\dot{\gamma}_{\mathrm{p}, \mathrm{st}}=0$ for $\sigma_{0}=0$, and find that $\dot{\gamma}_{\mathrm{p}, \mathrm{st}}$ and $\sigma_{0}$ have the same signs otherwise. In other words, as soon as $\sigma_{0} \neq 0$, there is some degree of plastic deformation. Depending on the circumstances, this plastic deformation may be negligible or dominant, as will be shown in the examples below. This discussion of the stationary state illustrates that the plastic deformation is captured in the model indirectly through the rate of hopping from one periodic image to the next. We point out that the expression for $j_{\mathrm{st}}$ with $c\left(\sigma_{0}\right)$ given by Eq. $11 \mathrm{~b}$ is very similar to the famous escape rate calculated by Kramers (1940). Furthermore, it can be shown that $\dot{\gamma}_{\mathrm{p}, \mathrm{st}}\left(\sigma_{0}\right)$ is well described by a hyperbolic sine, $\dot{\gamma}_{\mathrm{p}, \mathrm{st}} \propto \sinh \left(\Gamma \sigma_{0} /(2 \theta)\right)$, under the condition that the distribution $p_{\text {st }}(\gamma)$ be well localized around the minimum of $\Phi(\gamma)$. If $\sigma_{0}$ is proportional to the stress 
$\sigma$ in a certain range of deformation rates, as is the case for the results shown in Fig. 2, then this result is in accord with the relation $\dot{\gamma}_{\mathrm{p}, \mathrm{st}}=\dot{\gamma}_{\mathrm{p}, 0}^{\mathrm{E}} \sinh \left(\sigma / \sigma_{0}^{\mathrm{E}}\right)$ of Eyring between the steady state shear rate $\dot{\gamma}_{\mathrm{p}, \mathrm{st}}$ and the shear stress $\sigma$ (Krausz and Eyring 1975), for an appropriate choice of the constants $\dot{\gamma}_{\mathrm{p}, 0}^{\mathrm{E}}$ and $\sigma_{0}^{\mathrm{E}}$.

In order to illustrate the mechanical response both in stationary and transient conditions in the remainder of this paper, specific choices are made for the interaction potential $\Phi(\gamma)$ and the other parameters of the model. The $\gamma$ interval is chosen as $\Delta_{\Gamma}=[-0.5,0.5]$ (i.e., $\Gamma=1$ ), and for the interaction potential between the crystal layers, we assume Eq. 4 with $\Phi_{0} / \theta=10$. The energy and time scales are fixed by $\theta=1$ and $\hat{\eta}=$ 1 , respectively. For these values, the simulations have shown that the distribution function is well localized for $\sigma_{0} \leq 20$, which is essential for the interpretation of the kinetic theory model. Well localized states distant from the domain boundary in conjunction with a lowprobability current across the boundary corresponds to

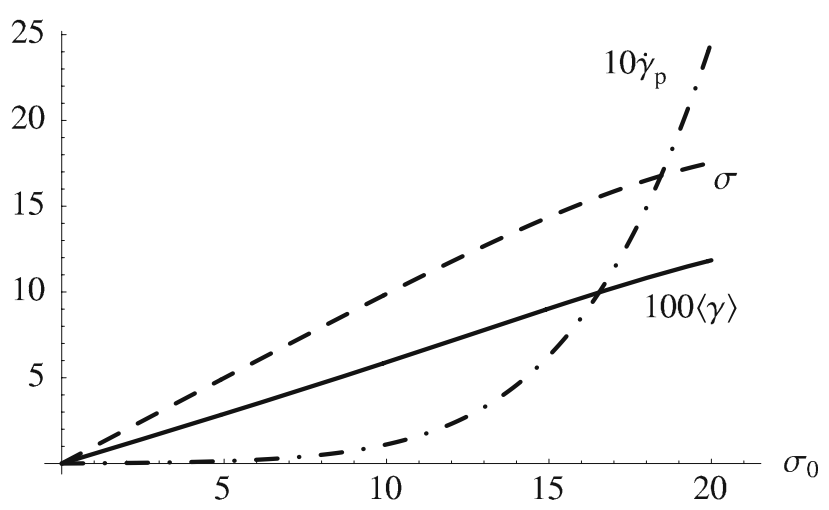

(a)

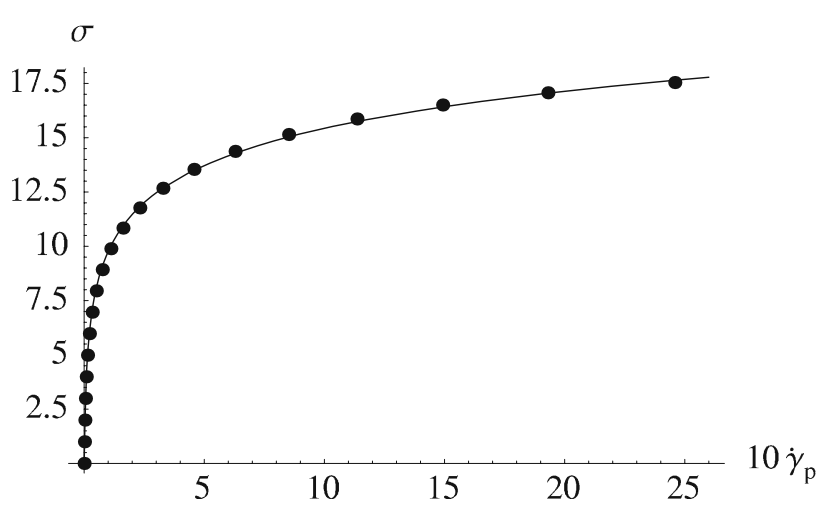

(b)

Fig. 2 a Steady state behavior of the model Eq. 6 as a function of the driving parameter $\sigma_{0}$ : average strain $\langle\gamma\rangle$ (solid), shear stress $\sigma$ (long dashed), plastic strain rate $\dot{\gamma}_{\mathrm{p}}$ (dashed-dotted). b Steady state shear stress $\sigma$ as a function of plastic strain rate $\dot{\gamma}_{\mathrm{p}}$ (solid line), with the dots representing the fit to the Eyring relation occasional hopping events between different equivalent states. In the following, we thus report results for $\sigma_{0} \leq$ 20.

For the stationary states according to Eq. 11, the results for $\langle\gamma\rangle, \sigma$, and $\dot{\gamma}_{\mathrm{m}}=\dot{\gamma}_{\mathrm{p}}$ are plotted in Fig. 2 . Since the relation between $\langle\gamma\rangle$ and $\sigma_{0}$ is almost linear up to $\langle\gamma\rangle=10 \%$, the stress-strain relation $\sigma(\langle\gamma\rangle)$ is easily read from Fig. 2a by scaling the $\sigma_{0}$-axis appropriately, leaving the shape of the shear stress curve almost unchanged. Figure $2 \mathrm{~b}$ shows the shear stress $\sigma$ as a function of the macroscopic strain rate $\dot{\gamma}_{\mathrm{m}}=\dot{\gamma}_{\mathrm{p}}$ with a finite slope of $\sigma$ at $\dot{\gamma}_{p}=0$. This stress-strain rate relation corresponds qualitatively to the experimental data for single-crystalline metals (see, e.g., Andrade and Henderson 1951). If read as $\dot{\gamma}_{\mathrm{p}}(\sigma)$, these data represent the stationary states of a creep test, indicative of soft yielding at $\sigma \simeq 10$. In order to connect to phenomenological relations frequently used in the literature, the flow curve $\sigma\left(\dot{\gamma}_{p}\right)$ has been fitted with the following two functions. First, the Eyring relation mentioned previously, which has also been used to characterize experimental data (e.g., see Gantchenko et al. 2008), was found to represent the model prediction well, as expected, see Fig. 2b. However, the second, more common choice, $\dot{\gamma}_{\mathrm{p}} \sim \sigma^{k}$, as applied, e.g., in Asaro (1983); Pan and Rice (1983), is inadequate to represent the behavior of the kinetic toy model.

\section{Transient situations}

Transient situations are examined by solving the diffusion equation (Eq. 6) numerically. In particular, prescribing the macroscopic shear rate $\dot{\gamma}_{\mathrm{m}}$ (Eq. 10b) as the control parameter requires a self-consistent solution strategy, since $\dot{\gamma}_{\mathrm{m}}$ depends on the solution $p(\gamma, t)$ itself. Figure 3 shows the response of the system under a constant macroscopic shear rate $\dot{\gamma}_{\mathrm{m}}=1.5$ over the time interval $[0,0.2]$. The results in terms of $\langle\gamma\rangle, \sigma$, and $\dot{\gamma}_{\mathrm{p}}$ represent the main features of experimental curves (Schmid and Boas 1935; Callister 2000; Haasen 1978). In the early stages $(t<0.02)$, one has $\dot{\gamma}_{\mathrm{m}}=d\langle\gamma\rangle / d t$ and the stress builds up accordingly. As time progresses $(0.02<t<0.1)$, one observes a smooth onset of yielding, i.e., the average $\langle\gamma\rangle$ saturates and plastic slip sets in until $\dot{\gamma}_{\mathrm{m}}=\dot{\gamma}_{\mathrm{p}}$, followed by an extended easy glide regime. The similarity in the time-dependence of $\sigma$ and $\langle\gamma\rangle$ is expressed in their ratio, which changes only slightly over the course of time, from $\sigma /\langle\gamma\rangle \simeq 175$ to $\sigma /\langle\gamma\rangle \simeq 150$.

Oscillatory deformations with imposed macroscopic shear rate $\dot{\gamma}_{\mathrm{m}}=\dot{\gamma}_{\mathrm{m}, 0} \cos \left(2 \pi t / t_{0}\right)$ have been studied for different maximum shear rates $\dot{\gamma}_{\mathrm{m}, 0}$ and cycle times $t_{0}$. In order to interpret the results, we recall that 


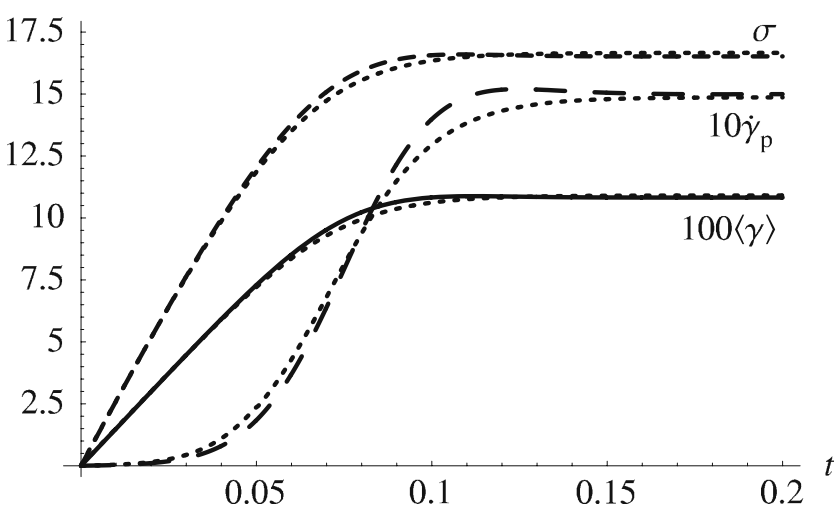

Fig. 3 Transient behavior of the model Eq. 6 at constant macroscopic strain rate $\dot{\gamma}_{\mathrm{m}}=1.5$ as a function of time $t$ : average strain $\langle\gamma\rangle$ (solid), shear stress $\sigma$ (short-dashed), plastic strain rate $\dot{\gamma}_{\mathrm{p}}$ (long-dashed). For comparison, the behavior of the closed firstmoment equation is shown (dotted)

the characteristic diffusion time is unity for the above choice of parameters, and the maximum strain is given by $\gamma_{\max }=\dot{\gamma}_{\mathrm{m}, 0} t_{0} /(2 \pi)$. Figure 4 shows that, even for a cycle time equal to the characteristic diffusion time, the hysteresis is small if the maximum strain does not allow for escape into another energy well, i.e., $\gamma_{\max } \ll \Gamma / 2$. In contrast, for $\dot{\gamma}_{\mathrm{m}, 0}=3$, one observes $\gamma_{\max } \simeq \Gamma / 2$, and hence, plastic slip occurs with substantial hysteresis. When increasing $\dot{\gamma}_{\mathrm{m}, 0}$ further (not shown here), the stress levels reached during the cycle increase only weakly with increasing strain rate, in accord with Fig. 2 and with corresponding systematic experimental studies (Estrin et al. 1996). Figure 5 shows two simulations with the same maximum strain rate but different cycle times, such that $\gamma_{\max } \ll \Gamma / 2$ and $\gamma_{\max } \simeq \Gamma / 2$, respectively. While the former conditions lead to (close to) reversible deformation, the latter bring about large

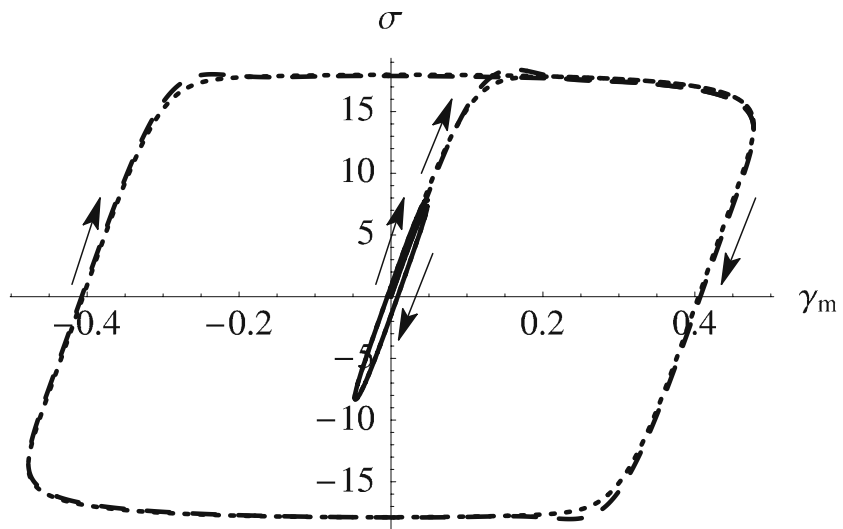

Fig. 4 Shear stress as a function of macroscopic strain $\gamma_{\mathrm{m}}=$ $\int_{0}^{t} \dot{\gamma}_{\mathrm{m}} d t$ of the model Eq. 6 under cyclic loading with imposed macroscopic shear rate $\dot{\gamma}_{\mathrm{m}}=\dot{\gamma}_{\mathrm{m}, 0} \cos \left(2 \pi t / t_{0}\right)$ for $t_{0}=1$. Solid line, $\dot{\gamma}_{\mathrm{m}, 0}=0.3$; dashed line, $\dot{\gamma}_{\mathrm{m}, 0}=3$. For comparison, the behavior of the closed first-moment equation is shown (dotted)

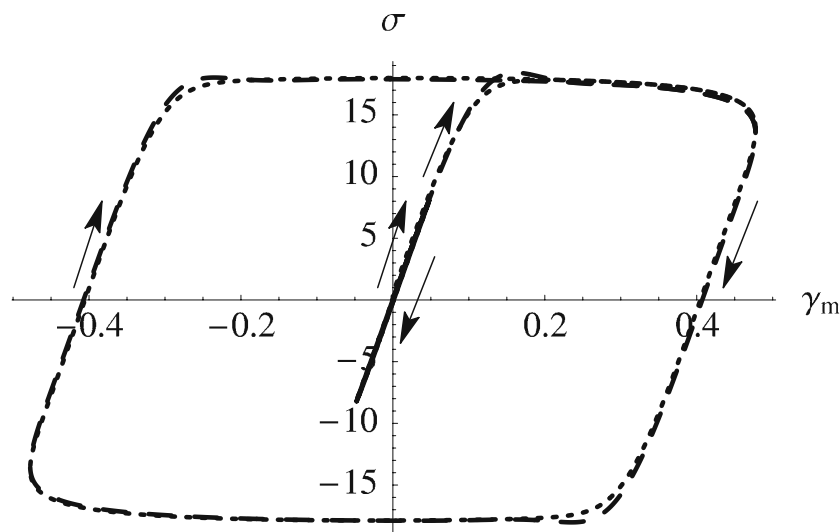

Fig. 5 Shear stress as a function of macroscopic strain $\gamma_{\mathrm{m}}=$ $\int_{0}^{t} \dot{\gamma}_{\mathrm{m}} d t$ of the model Eq. 6 under cyclic loading with imposed macroscopic shear rate $\dot{\gamma}_{\mathrm{m}}=\dot{\gamma}_{\mathrm{m}, 0} \cos \left(2 \pi t / t_{0}\right)$ for $\dot{\gamma}_{\mathrm{m}, 0}=3$. Solid line, $t_{0}=0.1$; dashed line, $t_{0}=1$. For comparison, the behavior of the closed first-moment equation is shown (dotted)

plastic deformations. Upon increasing $t_{0}$ further (not shown here), the stress levels reached during the cycle increase only weakly with increasing cycle time, again in accord with experimental studies (Estrin et al. 1996).

\section{Reduction to a closed first-moment description}

The kinetic toy model discussed above shall now be reduced to a closed-form evolution equation for the average elastic strain, $\langle\gamma\rangle$. In order to interpret Eq. $10 \mathrm{~b}$ as an evolution equation for $\langle\gamma\rangle$ for given $\dot{\gamma}_{\mathrm{m}}$, one needs to close the equation by expressing the plastic strain rate $\dot{\gamma}_{\mathrm{p}}$ defined in Eq. 8 in terms of $\langle\gamma\rangle$ and $\dot{\gamma}_{\mathrm{m}}$. To that end, in turn, it is necessary to choose an approximation to the true distribution function that is parametrized by the average $\langle\gamma\rangle$. As we have seen when discussing the stationary states of the full kinetic model in the section "Stationary situations," $\langle\gamma\rangle$ can be mapped to $\sigma_{0}$. Therefore, the stationary distribution in Eq. 11 can be parameterized effectively by $\langle\gamma\rangle$. Conclusively, an attractive procedure towards finding a closed evolution equation for the average elastic strain consists in using the manifold of stationary states as approximate distribution functions for transient situations. This ansatz for the approximate distribution function can also be motivated by the maximum entropy principle (Ilg et al. 2002, 2003; Gorban and Karlin 2005), also known as a quasi-equilibrium approach or generalized canonical ensemble, as shown in the following.

We start with defining the entropy $S$ of the distribution $p(\gamma)$ as

$S[p]=-k_{\mathrm{B}} \int_{-\Gamma / 2}^{\Gamma / 2} p(\gamma) \ln \left[p(\gamma) / p_{0}\right] d \gamma$, 
with the Boltzmann constant $k_{\mathrm{B}}$ and $p_{0}$ a constant to make the argument of the logarithm dimensionless. According to the maximum entropy principle, $S$ must be maximized under certain constraints. In the present case, those constraints include having control of the average energy $E$, of the normalization $N$, and most importantly, for studying out-of-equilibrium plastic flow, also of the average $\langle\gamma\rangle$,

$$
\begin{aligned}
& E[p]=\int_{-\Gamma / 2}^{\Gamma / 2} \Phi(\gamma) p(\gamma) d \gamma, \\
& N[p]=\int_{-\Gamma / 2}^{\Gamma / 2} p(\gamma) d \gamma=1, \\
& \bar{\gamma}[p]=\int_{-\Gamma / 2}^{\Gamma / 2} \gamma p(\gamma) d \gamma=\langle\gamma\rangle .
\end{aligned}
$$

Using the technique of Lagrange parameters to maximize $S$ under the conditions $E, N$, and $\bar{\gamma}$ leads to a solution that is proportional to $\exp (-\tilde{\Phi}(\gamma ; \alpha))$ with $\tilde{\Phi}$ defined prior to Eq. 11. Since this solution is not periodic for $\alpha \neq 0$, we use the same construction as in relation to Eq. 11 to arrive at

$p(\gamma ; \alpha)=\frac{1}{\mathcal{N}(\alpha)} e^{-\tilde{\Phi}(\gamma ; \alpha)}\left[1-c(\alpha) \int_{-\Gamma / 2}^{\gamma} e^{\tilde{\Phi}\left(\gamma^{\prime} ; \alpha\right)} d \gamma^{\prime}\right]$,

with the function $c$ defined in Eq. 11b.

In other words, the maximum entropy principle leads to a form of the distribution function that collapses with the true distribution function under stationary conditions. In view of Fig. 2a for the stationary solution and upon replacing $\sigma_{0}$ by $\alpha$, it is thus clear that the parameter $\alpha$ can be used to control the average $\langle\gamma\rangle$, and vice versa. This, in turn, means that, by employing the approximation Eq. 13, one can determine $\alpha=$ $\alpha(\langle\gamma\rangle)$, and hence, also $c=c(\langle\gamma\rangle), \mathcal{N}=\mathcal{N}(\langle\gamma\rangle)$, and $\sigma=\sigma(\langle\gamma\rangle)$. As a final step for bringing Eq. 10b into the form of a closed evolution equation for $\langle\gamma\rangle$, we express the plastic strain rate (Eq. 8) as

$$
\begin{aligned}
\dot{\gamma}_{\mathrm{p}}\left(\dot{\gamma}_{\mathrm{m}},\langle\gamma\rangle\right)= & \frac{\Gamma}{\mathcal{N}(\alpha)}\left(\dot{\gamma}_{\mathrm{m}}+\frac{\sigma-\alpha}{\hat{\eta}}\right) e^{-\tilde{\Phi}(-\Gamma / 2 ; \alpha)} \\
& +\frac{\Gamma D c(\alpha)}{\mathcal{N}(\alpha)}
\end{aligned}
$$

where we have made use of the relation Eq. 10a for $\sigma_{0}$. This form of $\dot{\gamma}_{\mathrm{p}}$ can indeed be expressed exclusively in terms of $\dot{\gamma}_{\mathrm{m}}$ and $\langle\gamma\rangle$ after invoking the relations $\alpha=\alpha(\langle\gamma\rangle)$ and $\sigma=\sigma(\langle\gamma\rangle)$. Note that the second term on the right-hand side (r.h.s.) of Eq. 14 has the same form as what was discussed in relation to the stationary states in the section "Stationary situations." Therefore, the first term on the r.h.s. is present only in transient situations and vanishes for $\alpha=\sigma+\hat{\eta} \dot{\gamma}_{\mathrm{m}}$, which is equal to $\sigma_{0}$.

The behavior of the reduced $\langle\gamma\rangle$ description (Eqs. 10b and 14) can be discussed in two parts. First, the stationary behavior must collapse with the full kinetic toy model of the section "Formulation of the kinetic toy model" since the parametrized approximate distribution function (Eq. 13) is identical to the exact stationary solution of the full kinetic model. Second, we address the transient behavior of the reduced description. The dotted lines in Figs. 3-5 show clearly that the reduced description compares well with the full kinetic model. Certainly, all stationary data must coincide between the two descriptions; however, also in the transient situations, the form Eq. 13 seems to be a reasonable approximation to the true distribution function.

The dependence of the plastic strain rate $\dot{\gamma}_{p}$ on the (total) strain rate $\dot{\gamma}_{\mathrm{m}}$ warrants special attention. In macroscopic theories of elasto-viscoplasticity (Boyce et al. 1988; Tervoort et al. 1998; Hütter and Tervoort $2008 \mathrm{~b}$ ), it is assumed that the plastic deformation gradient $\kappa^{\mathrm{p}}$ depends on the state of deformation through the Cauchy stress tensor, but a dependence on the velocity gradient $\boldsymbol{\kappa}$ is usually absent. The reduction of the simple kinetic toy model above to a closed evolution equation for $\langle\gamma\rangle$ indicates that this point may have to be reconsidered. The effect of $\dot{\gamma}_{\mathrm{m}}$ on the plastic rate $\dot{\gamma}_{\mathrm{p}}$ is also demonstrated in Fig. 6. Not only is there a hysteresis in the $\dot{\gamma}_{\mathrm{p}}(\sigma)$-relation for the full kinetic toy model, but also for the reduced description, the plastic strain rate is not a function of the stress (or average strain) only. Conversely, when neglecting the first term on the r.h.s.

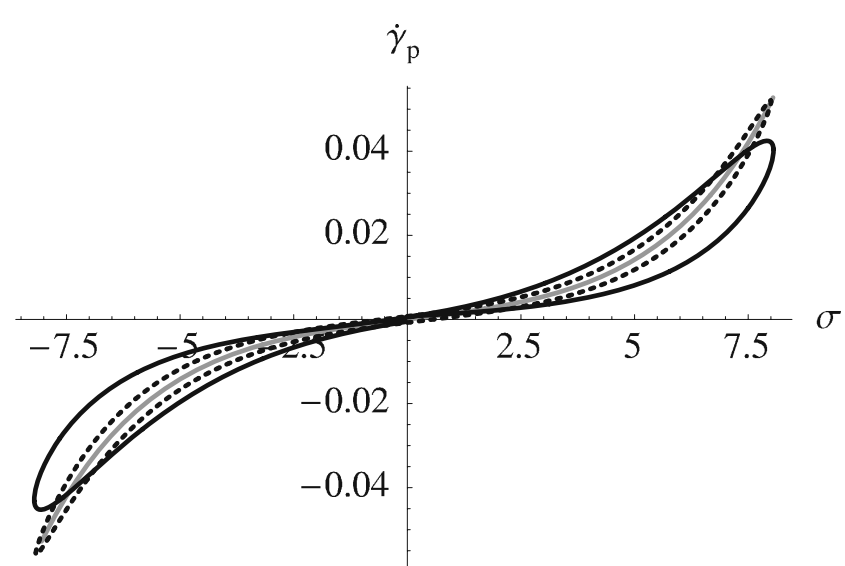

Fig. 6 Plastic strain rate $\dot{\gamma}_{p}$ as a function of shear stress $\sigma$ during cyclic loading with imposed macroscopic shear rate $\dot{\gamma}_{\mathrm{m}}=$ $\dot{\gamma}_{\mathrm{m}, 0} \cos \left(2 \pi t / t_{0}\right)$ for $\dot{\gamma}_{\mathrm{m}, 0}=3$ and $t_{0}=0.1$. Solid line, full $p(\gamma)-$ description; dotted line, $\langle\gamma\rangle$-model; gray line, $\langle\gamma\rangle$-model with neglect of the first term on the r.h.s. of Eq. 14 
of Eq. 14, i.e., neglecting the $\dot{\gamma}_{\mathrm{m}}$-dependence of $\dot{\gamma}_{\mathrm{p}}$ as done in common approaches in the literature (see the section "Introduction"), there is no hysteresis in the relation $\dot{\gamma}_{\mathrm{p}}(\sigma)$, as shown by the gray curve in Fig. 6 .

\section{Discussion}

The kinetic toy model introduced above accounts for both elastic (reversible) and plastic (irreversible) dynamics in the deformation of crystalline metals. Particularly, we focus on the conceptual question of how the irreversible plastic deformation emerges from the microscopic kinetics. The distinct origin of elastic and plastic rates of deformation has been clearly highlighted. Specifically, the periodicity in strain space represents the indistinguishability of reference states. The rate of transition between equivalent discrete reference states is captured by the plastic strain rate $\dot{\gamma}_{\mathrm{p}}$ in Eq. 8, as derived from the kinetic model, which is a major result of the paper. The mechanical response has been studied under stationary, start-up, and cyclic deformations. Particularly, Eyring's well-known result for stationary flows is recovered from the detailed kinetic toy model, while, notably, the latter allows one to also examine transient situations in detail. Furthermore, the kinetic toy model for the distribution of strains, $p(\gamma)$, has been reduced to a closed evolution equation for the average strain $\langle\gamma\rangle$. Doing so, we have shown that the plastic strain rate does not only depend on the shear stress, but also on the applied macroscopic shear rate. This hints at the possibility that, in tensorial models for elastoviscoplasticity, the plastic deformation gradient $\kappa^{\mathrm{p}}$ depends both on the Cauchy stress $\sigma$ and on the velocity gradient $\kappa$. Such possibilities must be on trial in future modeling efforts by using nonequilibrium thermodynamics techniques (Öttinger 2005; Hütter and Tervoort 2008b). From an experimental perspective, the examination of the relation $\dot{\gamma}_{p}(\sigma)$ under cyclic deformation should show fingerprints of such explicit effect of the applied shear rate.

The model, in both its full and reduced form, relates to more macroscopic descriptions of rate-dependent plasticity in three ways, similar to the use of the beadspring model in polymeric theory in conjunction with the hydrodynamic equations (Bird et al. 1987; Larson 1999; Öttinger 1996; Kröger 2005). Firstly, the shear stress $\sigma$ (Eq. 9) enters into the momentum balance, and secondly, the driving parameter $\sigma_{0}$ can be expressed purely in terms of the macroscopic quantities velocity gradient $\dot{\gamma}_{\mathrm{m}}$ and shear stress $\sigma$, according to Eq. 10a (see also Öttinger, unpublished manuscript). Thirdly, the average $\langle\gamma\rangle$ can be interpreted as the elastic part of the macroscopic deformation gradient (Boyce et al. 1988; Tervoort et al. 1998; Hütter and Tervoort 2008b), the relaxation of which is here resolved in terms of the kinetic model.

The kinetic toy model was designed to describe the physics of sliding crystal layers, and interesting conclusions have been derived from it. We wish to comment only briefly on how this model can be related to the picture of dislocations (Orowan 1934; Von Polanyi 1934; Taylor 1934). For reasons of simplification, we restrict ourselves to straight parallel dislocation lines. Since dislocation lines are made of a large number of atoms, as an entity, they feel the thermal Brownian motion. Therefore, the above toy model can be interpreted as describing the motion of a single dislocation line through the regular lattice. By a scaling argument it can be shown that the macroscopically observed strain $\gamma_{\text {macro }}$ is related to the microscopic strain around the single dislocation $\gamma_{\text {disl }}$ through $\gamma_{\text {macro }} \propto \rho \gamma_{\text {disl }}$ with $\rho$ the area density of dislocations. This relation between the kinetic toy model and the dislocation dynamics represents a modified form of the well-known Orowan relation (Asaro 1983; El-Azab 2000; Groma et al. 2003; Arsenlis et al. 2004). It is needless to say that such arguments must fall short of explaining strain-hardening due to the mutual interaction of dislocations, which notably was not the goal of this contribution. To aid in this respect, we envision that the techniques from nonequilibrium thermodynamics with the corresponding systematic coarse-graining techniques are useful.

It is well possible that not only materials with almostperfect crystalline order, as metallic single crystals, can be described by the kinetic toy model. Speculations in this direction are supported by the fact that we found approximately a hyperbolic sine relation between the plastic strain rate and the applied stress in steady states (see Fig. 2b), which is in accord with the description of Eyring and with experimental data on amorphous solid polymers (Bauwens-Crowet et al. 1969; Ward 1983). In this respect, it seems that the conclusions drawn in this paper are relevant whenever (1) rare hopping events occur under an applied mechanical load and (2) the favored microscopic configurations before and after each transition are very similar, i.e., "equivalent."

Acknowledgement We acknowledge useful discussions with Patrick Ilg.

\section{References}

Andrade END, Henderson C (1951) The mechanical behaviour of single crystals of certain face-centered cubic metals. Philos Trans R Soc S-A 244(880):177-203 
Arsenlis A, Parks D, Becker R, Bulatov VV (2004) On the evolution of crystallographic dislocation density in nonhomogeneously deforming crystals. J Mech Phys Solids 52:1213-1246

Asaro RJ (1983) Micromechanics of crystals and polycrystals. In: Hutchinson JW, Wu TY (eds) Advances in applied mechanics, vol 23. Academic, New York, pp 1-115

Bauwens-Crowet C, Bauwens JC, Homès G (1969) Tensile yieldstress behavior of glassy polymers. J Polym Sci A-2 Polym Phys 7:735-742

Beris AN, Edwards BJ (1994) Thermodynamics of flowing systems with internal microstructure. Oxford University, Oxford

Besseling JF, van der Giessen E (1993) Mathematical modelling of inelastic deformation (Applied mathematics and mathematical computation, vol 5). Chapmann and Hall, London

Bird BR, Curtiss CF, Armstrong RC, Hassager O (1987) Dynamics of polymeric liquids, vol 2: kinetic theory (2nd ed). Wiley, New York

Boyce MC, Parks DM, Argon AS (1988) Large inelastic deformation of glassy polymers. 1. rate dependent constitutive model. Mech Mater 7:15-33

Callister Jr WD (2000) Materials science and engineering: an introduction. Wiley, New York

El-Azab A (2000) Statistical mechanics treatment of the evolution of dislocation distributions in single crystals. Phys Rev B 61:11956-11966

Estrin Y (1996) Dislocation density-related constitutive modeling. In: Krausz AS, Krausz K (eds) Unified constitutive laws of plastic deformation. Academic, San Diego, pp 69-106

Estrin Y, Braasch H, Brechet Y (1996) A dislocation density based constitutive model for cyclic deformation. J Eng Mater Technol ASME 118:441-447

Gantchenko V, Jouinot P, Koster A (2008) Viscoplastic behaviour of metals deformed at high-temperature, application to tension-compression cycles. J Mater Sci 43:5342-5349

Gardiner CW (1985) Handbook of stochastic methods for physics, chemistry and the natural sciences (2nd ed). Springer, Berlin

Gorban AN, Karlin IV (2005) Invariant manifolds for physical and chemical kinetics. Lecture notes in physics, vol 660. Springer, Berlin

Grmela M, Öttinger HC (1997) Dynamics and thermodynamics of complex fluids. I. Development of a general formalism. Phys Rev E 56:6620-6632

Groma I, Csikor FF, Zaiser M (2003) Spatial correlations and higher-order gradient terms in a continuum description of dislocation dynamics. Acta Mater 51:1271-1281

Haasen P (1978) Physical metallurgy. Cambridge University Press, Cambridge

Hutchinson JW (1976) Bounds and self-consistent estimates for creep of polycrystalline materials. Proc R Soc Lond A Mat 348:101-127

Hütter M, Tervoort TA (2008a) Finite anisotropic elasticity and material frame indifference from a nonequilibrium thermodynamics perspective. J Non-Newton Fluid Mech 152: $45-52$

Hütter M, Tervoort TA (2008b) Thermodynamic considerations on non-isothermal finite anisotropic elasto-viscoplasticity. J Non-Newton Fluid Mech 152:53-65

Hütter M, Tervoort TA (2008c) Coarse graining in elastoviscoplasticity: Bridging the gap from microscopic fluctuations to dissipation. In: van der Giessen E, Aref $\mathrm{H}$ (eds) Advances in applied mechanics, vol 42. Academic, New York, pp 253-317
Ilg P, Karlin IV, Öttinger HC (2002) Canonical distribution functions in polymer dynamics: (I). Dilute solutions of flexible polymers. Physica A 315:367-385

Ilg P, Karlin IV, Kröger M, Öttinger HC (2003) Canonical distribution functions in polymer dynamics: (II). Liquid-crystalline polymers. Physica A 319:134-150

Kocks UF (1976) Laws for work-hardening and low-temperature creep. J Eng Mater Technol ASME 98:76-85

Kocks UF, Mecking H (2003) Physics and phenomenology of strain hardening: the FCC case. Prog Mater Sci 48:171-273

Kramers HA (1940) Brownian motion in a field of force and the diffusion model of chemical reactions. Physica (Utrecht) 7:284-304

Krausz A, Eyring H (1975) Deformation kinetics. WileyInterscience, London

Kröger M (2005) Models for polymeric and anisotropic liquids, Lect. Notes Phys. 675. Springer, Berlin

Kubo R (1966) Fluctuation-dissipation theorem. Rep Prog Phys 29:255-284

Kubo R, Toda M, Hashitsume N (1985) Statistical physics II: nonequilibrium statistical mechanics (2nd ed). Springer, New York

Kuhlmann-Wilsdorf D (1985) Theory of work-hardening 1934 1984. Metal Trans A 16:2091-2108

Larson RG (1999) The structure and rheology of complex fluids. Oxford University Press, New York

Leonov AI (1976) Nonequilibrium thermodynamics and rheology of viscoelastic polymer media. Rheol Acta 15:85-98

Mecking H, Kocks UF (1981) Kinetics of flow and strainhardening. Acta Metal 29:1865-1875

Orowan E (1934) Plasticity of crystals. Z Phys 89:605-659

Öttinger HC (1996) Stochastic processes in polymeric fluids: tools and examples for developing simulation algorithms (1st ed). Springer, Berlin

Öttinger HC, Grmela M (1997) Dynamics and thermodynamics of complex fluids. II. Illustrations of a general formalism. Phys Rev E 56:6633-6655

Öttinger HC (2005) Beyond equilibrium thermodynamics. Wiley, Hobroken

Pan J, Rice JR (1983) Rate sensitivity of plastic-flow and implications for yield-surface vertices. Int J Solids Struct 19:973-987

Rubin MB (1994) Plasticity theory formulated in terms of physically based microstructural variables part 1 . Theory. Int J Solids Struct 31:2615-2634

Rubin MB (1996) On the treatment of elastic deformation in finite elastic-viscoplastic theory. Int J Plast 12:951-965

Schmid E, Boas W (1935) Kristallplastizität. Springer, Berlin

Schulze V, Vöhringer O (2001) Plastic deformation: constitutive description. In: Buschow KHJ et al. (eds) Encyclopedia of materials: science and technology, vol 7. Elsevier, Amsterdam, pp 7050-7064

Tabourot L, Fivel M, Rauch E (1997) Generalised constitutive laws for fcc single crystals. Mat Sci Eng A Struct 234:639_ 642

Taylor GI (1934) Plastic deformation of crystals. Proc R Soc Lond Sec A 145:362-404

Tervoort TA, Smit RJM, Brekelmans WAM, Govaert LE (1998) A constitutive equation for the elasto-viscoplastic deformation of glassy polymers. Mech Time-Dep Mater 1:269-291

Truesdell C, Noll W (1992) The non-linear field theories of mechanics (2nd ed). Springer, Berlin

Von Polanyi M (1934) Lattice distortion which originates plastic flow. Z Phys 89, 660-662

Ward IM (1983) Mechanical properties of solid polymers (2nd ed). Wiley, Chichester 